\title{
Submicron-fabrication of Bovine serum albumin (BSA) matrix via two-photon photochemical crosslinking
}

\author{
Jinye $\mathrm{Xu}$, Jinping Cheng, Shuk Han Cheng, Barbara.P.Chan* \\ Tissue Engineering Laboratory, Department of Mechanical Engineering \\ The University of Hong Kong, Hong Kong SAR, China \\ Department of Biology and Chemistry \\ City University of Hong Kong, Hong Kong SAR, China \\ bpchan@hkucc.hku.hk
}

\begin{abstract}
We present the successful fabrication of bovine serum albumin matrix with novel two-photon photochemical crosslinking technique. Electron scanning microscope images revealed the highly porous microstructures of as-fabricated matrices. Dose dependence of the microstructures on both BSA and Rose Bengal has been studied. 2D patterns and 3D micropillars of BSA have also been fabricated, which potentially can be used as a model of cell-matrix study.
\end{abstract}

Keywords-photochemial crosslinking, multi-photon absortion, micro-fabrication

\section{INTRODUCTION}

With the advances in materials processing technologies, a variety of engineered extracellular matrices were made with micro- or nano-scale features. It's been proposed that both physical and mechanical factors can contribute to affect the cell-matrix relations. Currently, researchers have been able to process synthetic polymer hydrogels with tunable mechanical compliance. Engler and coworkers [1] used polyacrylamide gels as substrates with controllable tissue-level elasticity and found stem cells are quite sensitive to the elasticity. However, such a tuning is far from the micro-scale. It's been demonstrated that micro- and nano-scale geometries can be sensed by stem cells by neurite outgrowth [2] and cell alignment [3]. Although the exact mechanisms are unknown, both of the elasticity and geometry sensitivity of stem cells are related to cytoskeletal organizations. In addition, focal adhesions, the physical sensing components of cells are laterally on micrometer square scale [4]. Therefore it will be both interesting and promising to establish such a platform that allows micro-scale control of mechanical properties.

Comparing with synthetic polymers, which are easier to control in terms of patterning or tuning mechanical properties, biomaterials (e.g. collagen) have more advantages such as biocompatibility but are more difficult to process. ECM protein-based hydrogels, such as collagen and fibrin, are often used in cell studies. However, it is a major challenge to improve and control the mechanical properties of biomaterials. Various crosslinking methods have been applied to overcome their weakness. In our lab, single-photon photochemical crosslinking of collagen has been demonstrated to be able to increase the physicochemical properties of collagen scaffolds and this effect is related to the modified microstructure of collagen scaffolds comparing with those without photochemical treatment [5]. This result indicates that photochemical crosslinking is an effective approach to enhance mechanical properties of biomaterials. By taking advantage of two-photon photochemical crosslinking, we expect to achieve micro-scale spatial control over mechanical properties of biomaterials.

Two-photon photochemical crosslinking is based on a multi-photon absorption (MPA) process, first predicted by Maria Goeppert-Mayer in 1931. In a MPA process, high intensity of illumination is required to allow the simultaneous absorption of two or three photons, the energies of which are combined to excite a chromophore from ground state to a higher electronic state. Unlike one-photon absorption, MPA is nonlinearly dependent on the illumination intensity, which makes it only happen at the focal volume. Polymerization and degradation of synthetic materials and photochemical crosslinking of proteins have been demonstrated upon twophoton or three-photon absorption.

In this study, we have demonstrated the fabrication of both 2D micro-patterns and 3D micro-structures using bovine serum albumin (BSA) as the protein substrate and rose Bengal (RB) as the photosensitizer. We have also investigated the dosage dependence of two-photon photochemical crosslinking process on the protein and the photosensitizer. This study provides a promising platform which can be potentially used to regulate stem cell fates by micro-scale control on both elasticity and geometry.

\section{MATERIALS AND METHODS}

\section{A. Materials}

In the current work, bovine serum albumin (Sigma) solutions containing the photochemical crosslinker rose Bengal (Sigma) were freshly prepared before the experiments. $30 \mu \mathrm{L}$ of the solution was dropped onto a coverglass and mounted on the 
working stage of an inverted multiphoton confocal laser scanning microscope.

Two types of multiphoton confocal laser scanning microscopes were used, Zeiss LSM 510 Meta and Leica TCS SP5, both of which were equipped with mode-locked Ti:sapphire femtosecond laser. $810 \mathrm{~nm}$ wavelength and $40 \times /$ oil lens were always used.

\section{B. Methods}

1) Fabrication of BSA matrices with two-photon photochemical crosslinking

By repeatedly scanning the laser spot in a line scan or frame scan mode within the solution, BSA can be crosslinked and deposited on the coverslip.

2) Dose effects of BSA and rose Bengal on the two-photon photochemical crosslinking

Single frame scan (no line average) with constant scanning speed and power were conducted in solutions containing a variety concentrations of $\mathrm{BSA}(50,25,12.5 \mathrm{mg} / \mathrm{ml})$ and $\mathrm{RB}$ $(0.1 \%, 0.05 \%, 0.025 \% \mathrm{w} / \mathrm{v})$.

3) Fabrication of user-defined $2 D$ protein patterns and $3 D$ micro-scale protein arrays.

Custom defined 2D patterns of BSA were fabricated by choosing the ROI (region of interest) function in the Zeiss LSM software during scanning.

3D micro-scale protein arrays were fabricated in a solution containing $66 \mathrm{mg} / \mathrm{ml}$ BSA and $0.1 \% \mathrm{w} / \mathrm{v} \mathrm{RB}$ by drawing multiple regions under the ROI function and doing a 'xyz' scan with Leica SP5. Every region was a square with $1.6 \mu \mathrm{m}$ in length. A total of 5 steps' scanning with the z-step size $2 \mu \mathrm{m}$ was conducted.

\section{4) Electron scanning microscopyanalysis}

After fabrication, electron scanning microscopy was used to evaluate microstructures of the proteins. Samples were fixed with $2.5 \%$ GTA for 20mins, washed in series ethanol-water solution (25\%-50\%-70\%-80\%-90\%-100\%), dehydrated by critical point drying, mounted on metal stages, sputter coated with gold for $100 \mathrm{~s}$ and imaged under electron scanning microscopy.

\section{RESULTS}

\section{A. Typical structures of BSA matrices fabricated by two-} photon photochemical crosslinking

Fig.1 shows a typical SEM image of a BSA matrix fabricated by repeatedly frame scanning. It is clear that within the field scanned by multiphoton laser, BSA was deposited on the coverglass. Higher magnification SEM picture reveals the highly porous microstructure of the adsorbed BSA matrix, which was composed of interconnected larger protein aggregates or discrete particles of smaller ones.

\section{B. Dose effects of BSA and rose Bengal on the two-photon photochemical crosslinking}
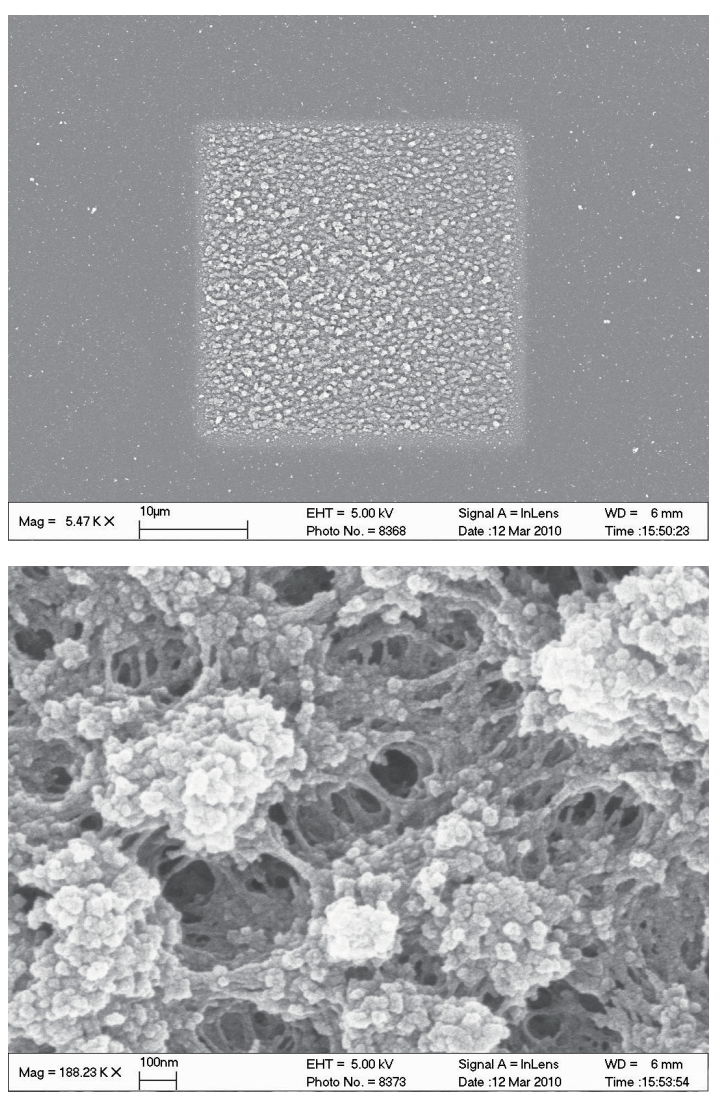

Fig. 1 SEM images of a typical BSA matrix

As shown in Fig.2, in the solution with higher concentrations of both BSA and RB $(50 \mathrm{mg} / \mathrm{mL}, 0.1$ and $0.05 \% \mathrm{w} / \mathrm{v})$, porous networks were fabricated, while with lower concentrations of either BSA (25 and $12.5 \mathrm{mg} / \mathrm{mL}$ ) or $\mathrm{RB}$ $(0.025 \% \mathrm{w} / \mathrm{v})$, only discrete particles were obtained.

\section{User-defined $2 D$ protein patterns and $3 D$ micro-scale protein arrays.}

A "HKU" pattern made with BSA is shown in Fig. 3. As shown in Fig.4, an array of 3D micropillars of BSA was fabricated. The height of the micropillar was about $2.1 \mu \mathrm{m}$, (Height $=1.376 / \sin 40^{\circ}=2.141 \mu \mathrm{m}, 40^{\circ}$ : rotation degree of the SEM stage)

\section{DISCUSSION}

This study has shown for the first time the ability of high throughput fabrication of 3D BSA microstructures. This is an efficient and highly reproducible fabrication technique. However, the heights of the BSA micropillars were much less than the total scanning height $(8 \mu \mathrm{m})$, which may due to the shrinkage during processing or intrinsic weakness of protein structures.

Dose dependence effect indicates that the concentrations of both protein and photosensitizer and below certain concentrations no matrices can be fabricated. 
This fabrication technique is very sensitive to the position where the laser spot is focused. Usually this position is chosen by raising the laser focal plane from under the coverglass until a sudden increase in fluorescence signals occurs. This position is hard to define especially when rose Bengal is of very low concentration.
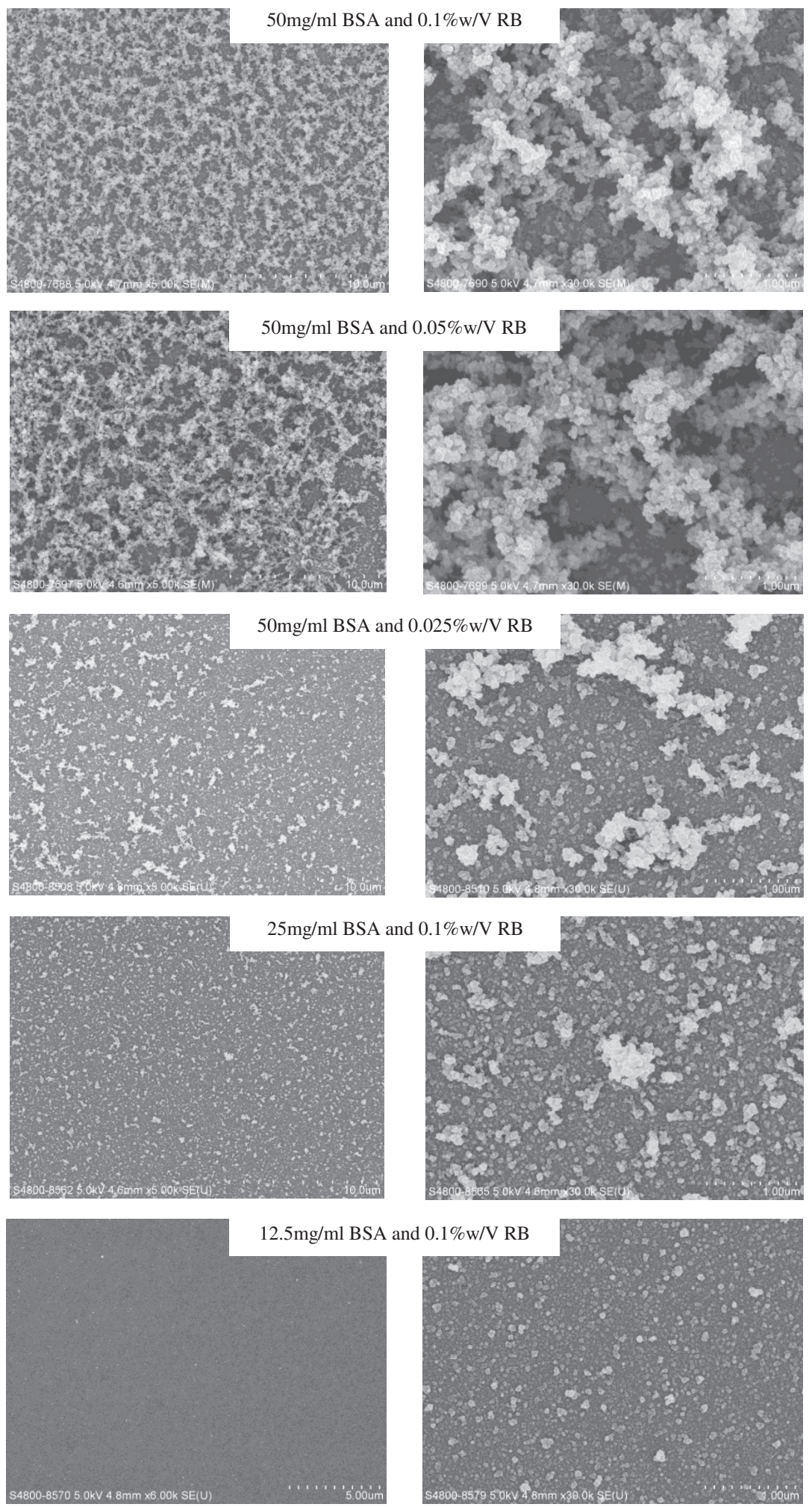

Fig 2. Low (left) and high (right) magnification SEM images of BSA matrices fabricated from solutions with different concentrations. 


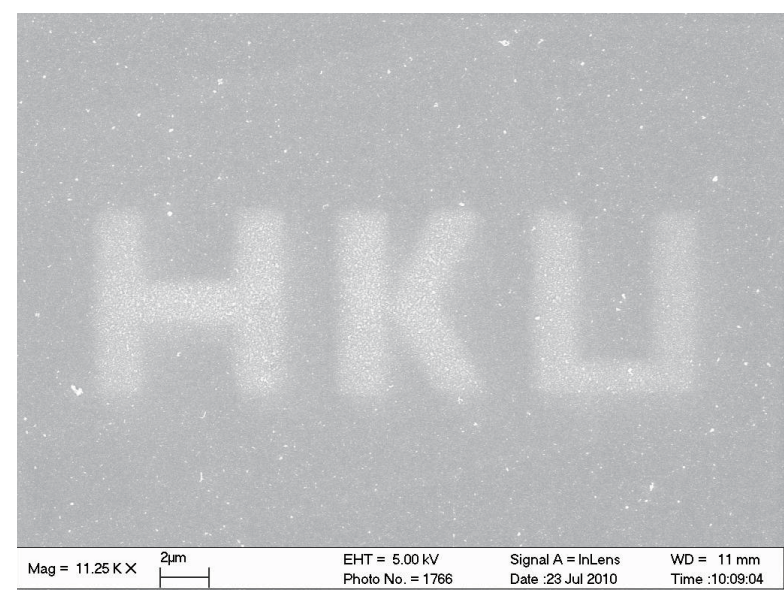

Fig.3 SEM image of a "HKU" pattern fabricated with BSA.

\section{CONCLUSION}

This study successfully fabricated 3D protein microstructure arrays using a novel two-photon photochemical crosslinking technique. This can be potentially used as a platform to study cell-matrix relations.

\section{ACKNOWLEDGMENT}

This work was supported by University Development Fund, Strategic Research Themes on Biomedical Engineering and Nanobiotechnology, HKU.

\section{REFENCES}

[1] Engler, A. J., S. Sen, et al. (2006). "Matrix Elasticity Directs Stem Cell Lineage Specification." Cell 126(4): 677-689.

[2] Hayman, M. W., K. H. Smith, et al. (2005). "Growth of human stem cell-derived neurons on solid three-dimensional polymers." Journal of Biochemical and Biophysical Methods 62(3): 231-240.

[3] Recknor, J. B., D. S. Sakaguchi, et al. (2006). "Directed growth and selective differentiation of neural progenitor cells on micropatterned polymer substrates." Biomaterials 27(22): 4098-4108.

[4] Balaban, N. Q., U. S. Schwarz, et al. (2001). "Force and focal adhesion assembly: a close relationship studied using elastic micropatterned substrates." Nat Cell Biol 3(5): 466-472.

[5] Chan, B. P. and K. F. So (2005). "Photochemical crosslinking improves the physicochemical properties of collagen scaffolds." J Biomed Mater Res A 75(3): 689-701.
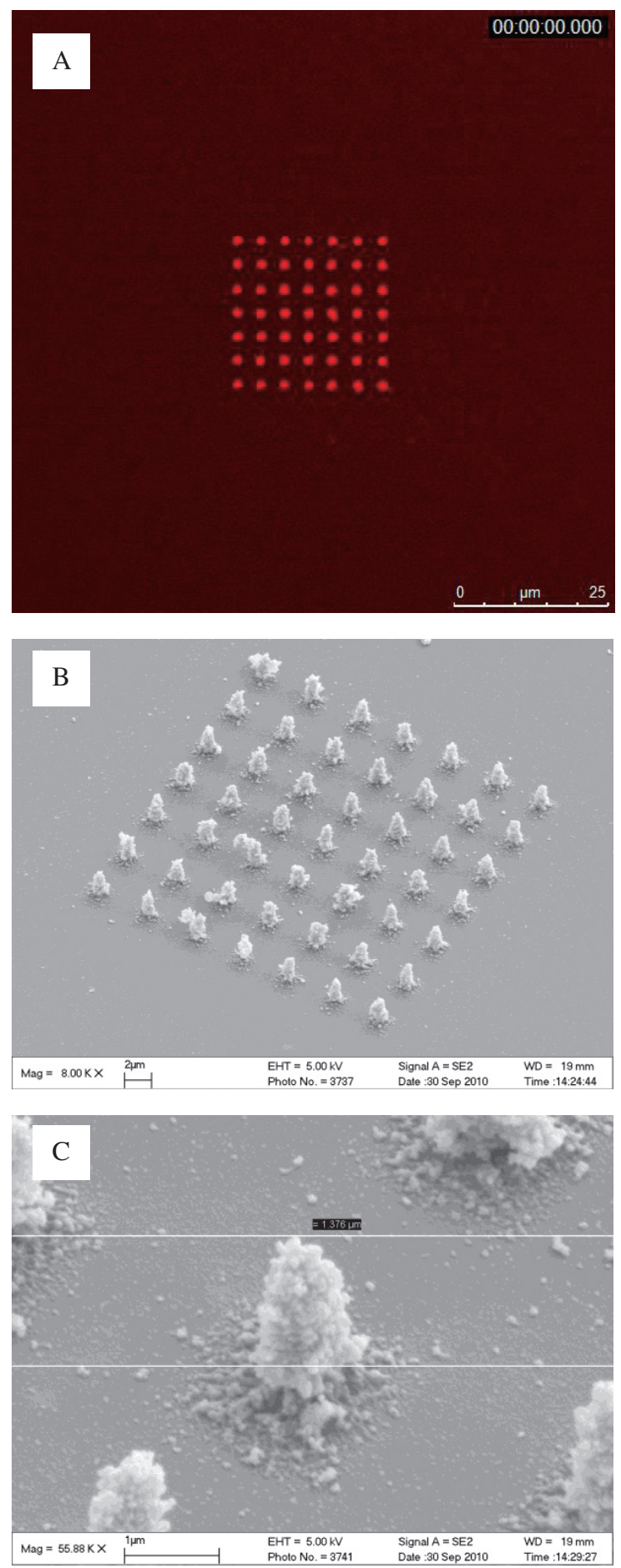

Fig.4 3D-micropillars of BSA: (A) Two-photon fluorescence image; (B) and (C) SEM images (tilted by $40^{\circ}$ ). 